Technical Progress on

\title{
InNOVATIVE EleCtromagnetic SENSORS FOR PIPELINE CRAWLERS
}

\author{
Type of Report: Technical Progress Report \\ Reporting Period Start Date: April 1, 2004 \\ Reporting Period End Date: September 30, 2004
}

by

J. Bruce Nestleroth

Submitted

November 5, 2004

NETL Award No. DE-FC26-03NT41881

PRCI Contract No. PR-003-03155

Battelle

505 King Avenue

Columbus, Ohio 43201 


\begin{abstract}
Notice
This report was prepared as an account of work sponsored by an agency of the United States Government. Neither the United States Government nor any agency thereof, nor any of their employees, makes any warranty, express or implied, or assumes any legal liability or responsibility for the accuracy, completeness, or usefulness of any information, apparatus, product, or process disclosed, or represents that its use would not infringe privately owned rights. Reference herein to any specific commercial product, process, or service by trade name, trademark, manufacturer, or otherwise does not necessarily constitute or imply its endorsement, recommendation, or favoring by the United States Government or any agency thereof. The views and opinions of authors expressed herein do not necessarily state or reflect those of the United States Government or any agency thereof.
\end{abstract}

Neither Battelle, nor any person acting on their behalf:

(1) Makes any warranty or representation, expressed or implied, with respect to the accuracy, completeness, or usefulness of any information contained in this report or that the use of any information, apparatus, method, or process disclosed in this report may not infringe privately owned rights.

(2) Assumes any liabilities with the respect to the use of, or for damages resulting from the use of any information, apparatus, method or process disclosed in this report. 


\begin{abstract}
Internal inspection of pipelines is an important tool for ensuring safe and reliable delivery of fossil energy products. Current inspection systems that are propelled through the pipeline by the product flow cannot be used to inspect all pipelines because of the various physical barriers they encounter. Recent development efforts include a new generation of powered inspection platforms that crawl slowly inside a pipeline and are able to maneuver past the physical barriers that can limit inspection. At Battelle, innovative electromagnetic sensors are being designed and tested for these new pipeline crawlers. The various sensor types can be used to assess a wide range of pipeline anomalies including corrosion, mechanical damage, and cracks.

The Applied Energy Systems Group at Battelle is concluding the first year of work on a projected three-year development effort. In this first year, two innovative electromagnetic inspection technologies were designed and tested. Both were based on moving high-strength permanent magnets to generate inspection energy. One system involved translating permanent magnets towards the pipe. A pulse of electric current would be induced in the pipe to oppose the magnetization according to Lenz's Law. The decay of this pulse would indicate the presence of defects in the pipe wall. This inspection method is similar to pulsed eddy current inspection methods, with the fundamental difference being the manner in which the current is generated. Details of this development effort were reported in the first semiannual report on this project.

This second semiannual report focuses on the development of a second inspection methodology, based on rotating permanent magnets. During this period, a rotating permanent magnet exciter was designed and built. The exciter unit produces strong eddy currents in the pipe wall. Our tests have shown that at distances of a pipe diameter or more, the currents flow circumferentially, and that these circumferential currents are deflected by pipeline defects such as corrosion and axially aligned cracks. Simple sensors are used to detect the change in current densities in the pipe wall.
\end{abstract}




\section{Table of Contents}

Page

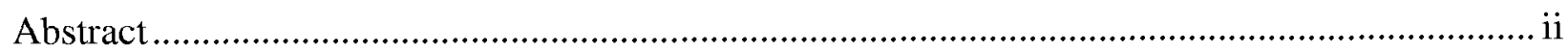

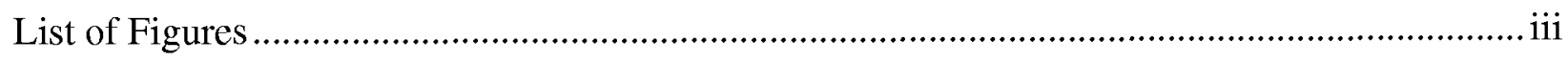

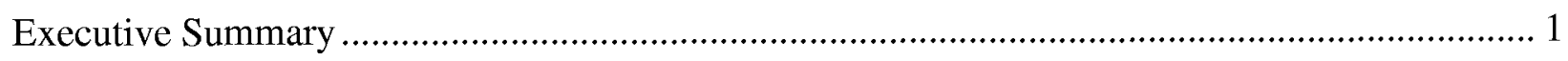

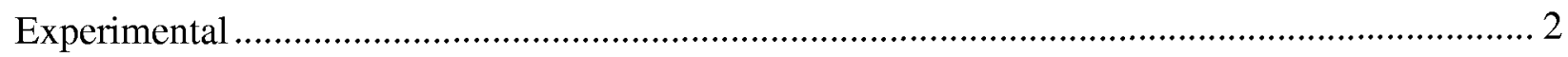

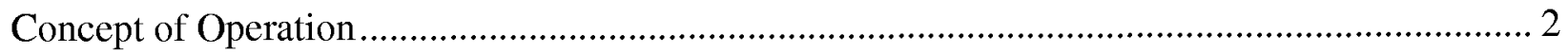

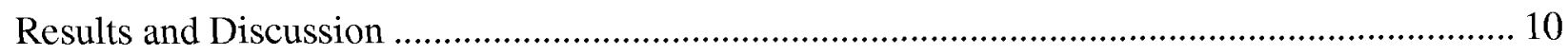

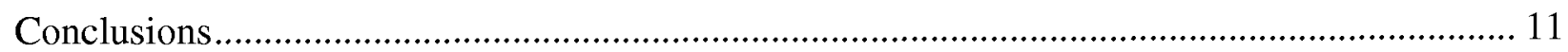

Appendix A - Pull Away Tests .................................................................................. A

Appendix B - Benchmark Tests at the Pipeline Simulation Facility .................................. B-1

\section{List of Figures}

Figure 1. Rotating permanent magnet exciter................................................................ 2

Figure 2. Prototype implementation of the rotating permanent magnet eddy current exciter....... 3

Figure 3. Two pole rotating permanent magnet exciter for 12 inch diameter pipe.................... 4

Figure 4. Finite-element modeling results for a two-pole magnetizer.................................. 5

Figure 5. Current flow as the magnetizer rotates in the pipe...............................................5

Figure 6. Axial and radial components of the magnetic field near the exciter (top) and one pipe

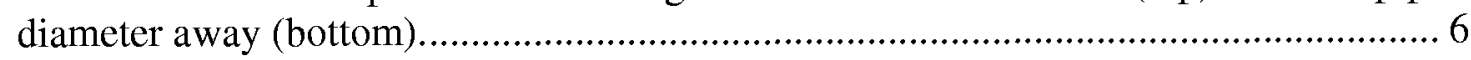

Figure 7. Axial decay of the currents flowing circumferential in the pipe wall....................... 7

Figure 8. Current strength for 3 magnetizer configurations. ........................................... 8

Figure 9. Typical signal from an area of pipe with a metal loss corrosion pit.......................... 9 


\section{Executive Summary}

The basic requirements for sensor systems for pipeline crawlers include small physical size and weight as well as low electrical power consumption. Magnetic flux leakage (MFL), the most common technology used to inspect pipelines, is difficult to implement on autonomous crawler systems because the sensors are large and heavy. In addition, although MFL sensors are slow moving, they require significant power to measure the flux leaking from defects and are not able to detect all defect types. Among alternative approaches, small and light sensor technologies have shown promise but implementation attempts have been thwarted by high speed and long distance requirements, factors that are not as restrictive for crawler-based inspection systems.

The objective of this project is to develop electromagnetic sensors for mounting on a crawling inspection platform that moves slowly through the pipeline interior. These sensors will be used to assess a wide range of pipeline conditions including corrosion (pitting, patches, and general), mechanical damage, cracking, and seam weld defects. The sensors must be light weight and low drag to minimize propulsion requirements of the crawler platform. In addition, the sensors will require minimal power for excitation of interrogating energy and sensor current for anomaly detection.

The focus of work in the first year of this project has been on prototype development. The first semiannual report covered the development of a translating permanent magnet induced pulsed eddy current system. The Applied Energy Systems Group at Battelle was successful in building a pulsed eddy current system that uses Hall Effect sensors to measure the decay of induced eddy currents for measuring wall thickness.

This second semiannual report covers the development of the rotating permanent magnet exciter to induce eddy currents for the inspection of pipelines. The Applied Energy Systems Group at Battelle built and tested a rotating permanent eddy current exciter that produces strong magnetic fields at the receiver and has the potential to use less electrical power than coil-based eddy current systems such as remote field eddy currents (RFEC). Currents were detectable with a simple Hall Effect sensor at distances up to three pipe diameters away. Simulated corrosion defects were detectable in a 12-inch diameter pipe with a wall thickness of 0.375 inches.

Also in this period, the Applied Energy Systems Group performed a benchtop implementation assessment of each system using similar defects. Since the results showed that the rotating system was more adaptable to pipeline inspection, only this system will be carried into the second year of the sensor development. 


\section{Experimental}

One approach to nondestructive inspection of materials involves injecting a uniform energy into an object. An anomaly or defect disrupts this uniformity. Sensors are used detect a change in the uniformity and thus the anomaly or defect. Radiography and magnetic flux leakage inspection are common examples of this approach. In radiography, anomalies are detected when more of the incident X-ray energy passes thru the material. A film or charge coupled device placed on the opposite side of the material as the source can be used to detect this change in absorption. In magnetic flux leakage, anomalies are detected when the uniform magnetic established in the material is disturbed. Magnetic field sensors such as Hall Effect sensors or moving coils detect the local changes magnetic fields due to anomalies. This second semiannual report covers work on the development of the rotating permanent magnet exciter to induce uniform eddy currents for the inspection of pipelines.

\section{Concept of Operation}

A new electromagnetic approach for pipes and tubes has been developed. The method uses rotating permanent magnets to produce an alternating electrical current flowing in the circumferential direction. Figure 1 is a cutaway drawing showing the in-pipe positioning of a rotating permanent magnet exciter, illustrating a concept that has the potential to induce strong eddy currents in the pipe wall. This approach uses alternating $\mathrm{N}$ and $\mathrm{S}$ poles rotating around a shaft, in contrast to traditional eddy current systems, which use a coaxial coil in the pipe that is driven by a sinusoidal current. The power needed by an exciting coil to produce a detectable signal can be significant. This may limit implementation to tethered systems, short-run crawlers, and systems with onboard power generation.

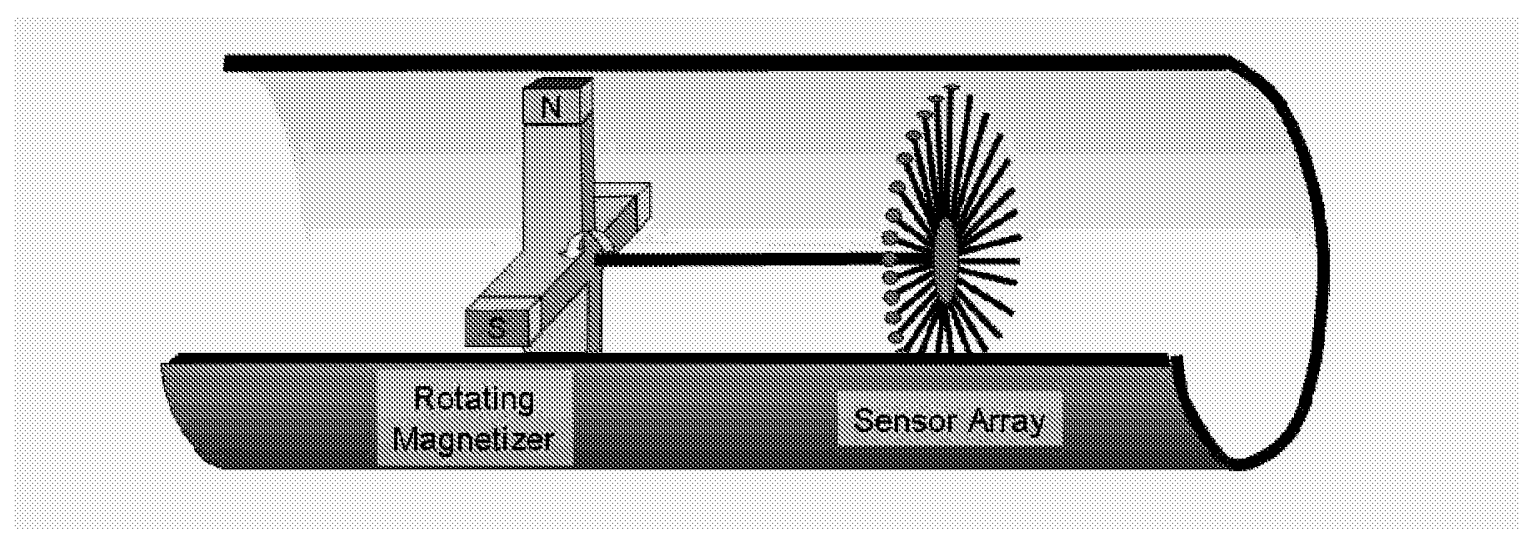

Figure 1. Rotating permanent magnet exciter.

\section{Development of the Initial Prototype}

The initial prototype implementation of the rotating permanent magnet exciter system is shown in Figure 2. The test specimen is a steel pipe sample $6.35 \mathrm{~mm}(0.25$ inch $)$ thick and $150 \mathrm{~mm}$ (6 inches) in diameter. Six neodymium-iron-boron $(\mathrm{NdFeB})$ magnets with an energy product of 
28 megagauss Oersted (MGOe) were used to generate eddy currents in the pipe. The cylindrical magnets were 1 inch $(25 \mathrm{~mm})$ in diameter and 0.5 inch $(12.7 \mathrm{~mm})$ thick. A variable speed direct current motor is used to rotate the magnetizing assembly. As reported in the previous semiannual report, the magnets produced a good field to the outside of the pipe under static conditions; however, the sinusoidal energy propagating through the pipe was less than optimal. It was concluded that enhancements to this configuration will be required to make this a viable inspection method.

The current in the pipe was detected using commercial linear Hall effect sensors, Honeywell Microswitch 495. The field levels were amplified by a factor of 100 using an operation amplifier after the Q-point offset was removed using a resistance voltage divider. A $1000 \mathrm{ohm}$ ten-turn potentiometer was used to adjust for the variation between sensors. Two sensors were collocated for the measurement of the axial and radial component of the magnetic field. Though repackaged in later inspection systems, this sensor system design was used on this initial prototype and the subsequent inspection system.

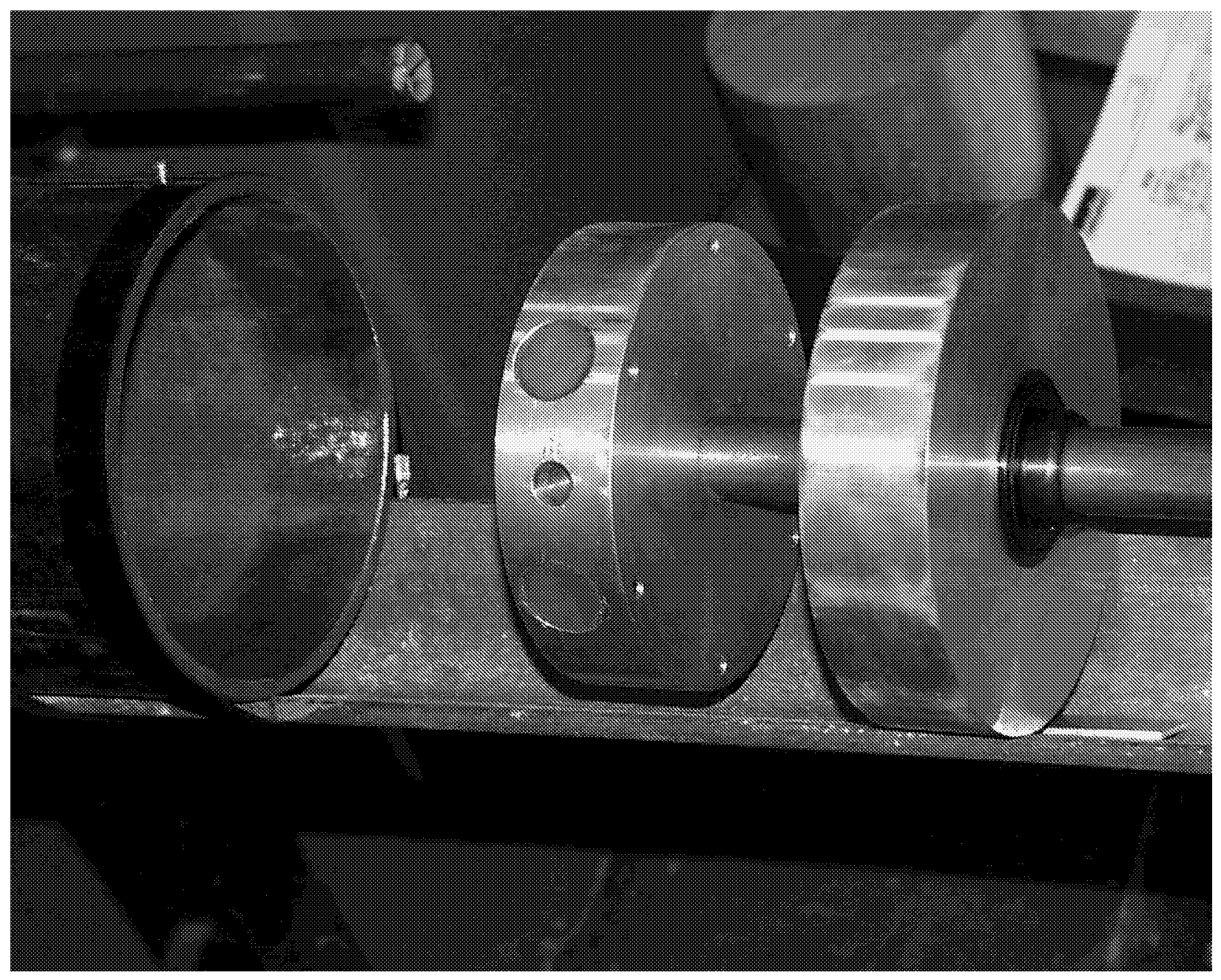

Figure 2. Prototype implementation of the rotating permanent magnet eddy current exciter. 


\section{Development of the Improved Prototype}

A combination of modeling and prototyping was used to improve the inspection current strength propagating along the pipe used to detect pipeline anomalies. A three-dimensional finiteelement model proved useful in understanding the current flow and improving the efficiency of current generation. After examining various configurations, the best performance was achieved using a simpler two-pole exciter. A prototype for a 12-inch diameter pipeline is shown in Figure 3. A pair of $\mathrm{NdFeB}$ magnets is placed on a steel core. The magnets are 2 inches long, 1 inch wide, and 0.5 inch thick; the magnet strength is 38 MGOe. The core was machined from 1018 steel. While the magnets have a strong attraction to the steel core, aluminum guide rails position the magnets precisely on the core. The air gap between the magnet and the 0.375 inch thick test pipe is 0.5 inches. Wheeled support plates keep the magnet centered in the pipe. A variable speed direct current motor is used to rotate the magnetizing assembly.

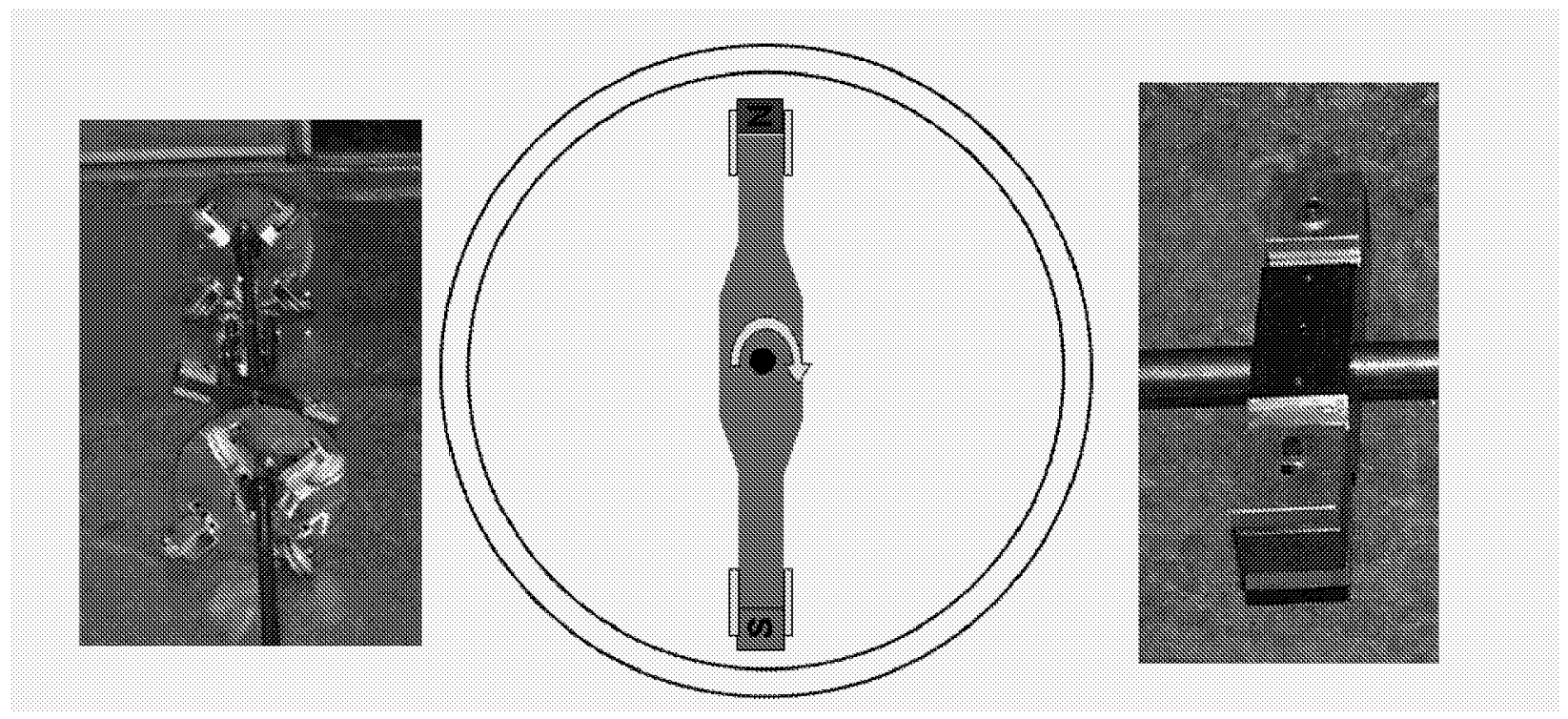

Figure 3. Two pole rotating permanent magnet exciter for 12 inch diameter pipe.

\section{Magnetic Finite Element Modeling}

Modeling shows that a two-pole magnetizer produces strong and uniform current densities at distances well away from the magnetizer. Figure 4 shows modeling results for the 12-inch prototype system rotating at $300 \mathrm{rpm}(5 \mathrm{~Hz})$. The calculations were performed using Vector Fields ${ }^{\circledR}$ three-dimensional rotational analysis solver. The image on the left shows the logarithm of the current magnitudes at the inside pipe wall. The current is strongest at the magnetizer poles, but becomes uniform at a distance of about half a pipe diameter away. The image on the right shows the current flow in the pipe, the direction as indicated by the arrows. While the current flow is complex near the rotating magnet poles, the current at a pipe diameter or more away from the magnetizer is uniform and sinusoidal. 

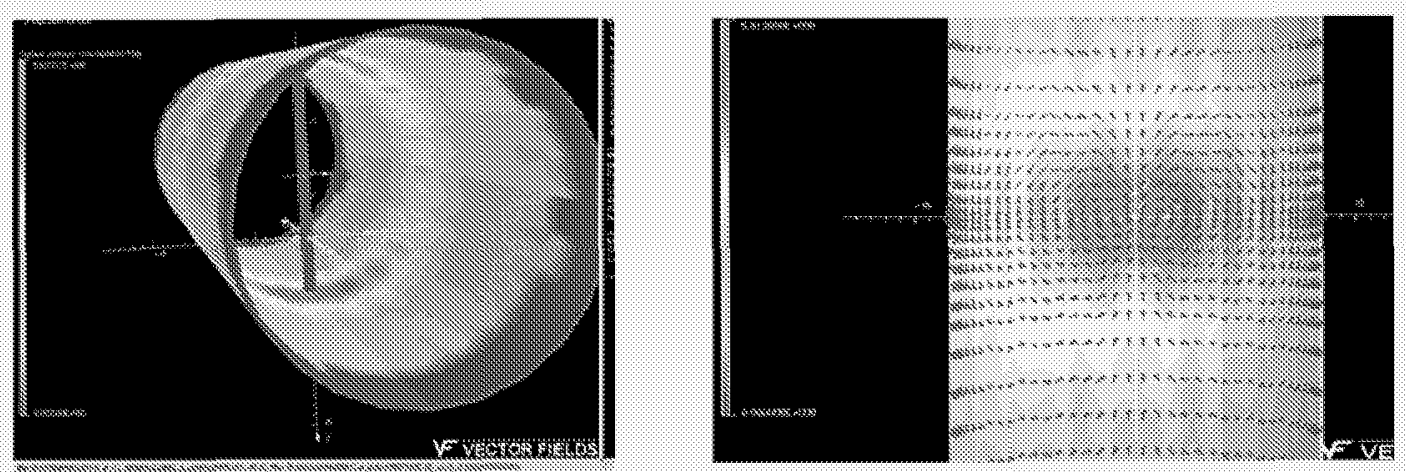

Figure 4. Finite-element modeling results for a two-pole magnetizer.

Figure 5 illustrates the current flow as the magnetizer rotates in the pipe. The current flows in an elliptical path around the magnets. When the magnetizer is vertical, strong currents flow axially along the sides of the pipe (top illustration) and circumferentially at the top and the bottom.

When the magnetizer is horizontal, strong currents flow circumferentially at the sides of the pipe and axially at the top and the bottom (second illustration). The direction of current flow depends on the polarity of the magnet and direction of rotation. The third and fourth illustrations show that the direction of current flow is opposite that of the first and second illustration because the magnet polarity has reversed.
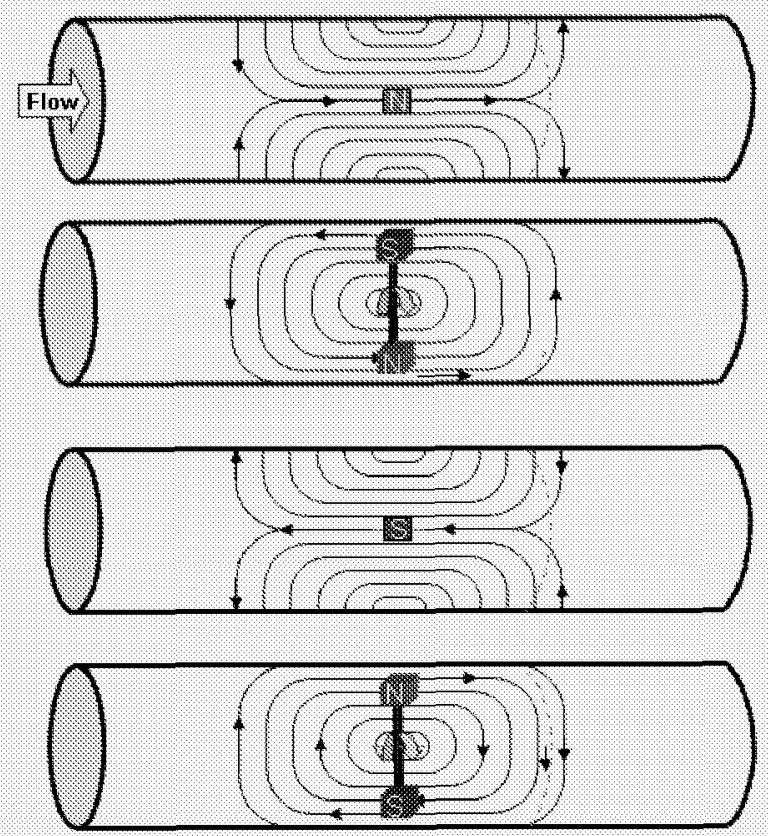

Figure 5. Current flow as the magnetizer rotates in the pipe. 


\section{Work Confirming Modeling Results}

The magnetic field in the pipe has two parts, each with distinct properties and effects. One part is the direct magnetic field from the strong permanent magnets. The second field is due to the current flowing in the pipe. Near the rotating magnets, the direct field from the magnet is dominant and produces a saddle-shaped alternating signal. Farther away from the magnets, magnetic field caused by the currents flowing in the pipe dominates. For a two-pole system in a 12 -inch pipe, the field due to direct field is negligible at distances greater than one pipe diameter (1D) and the measured signal is nearly sinusoidal. The field is also strong, on the order of a gauss. Appendix A contains results for other sensor to magnetizer separation distances and a four pole magnetizer configuration.
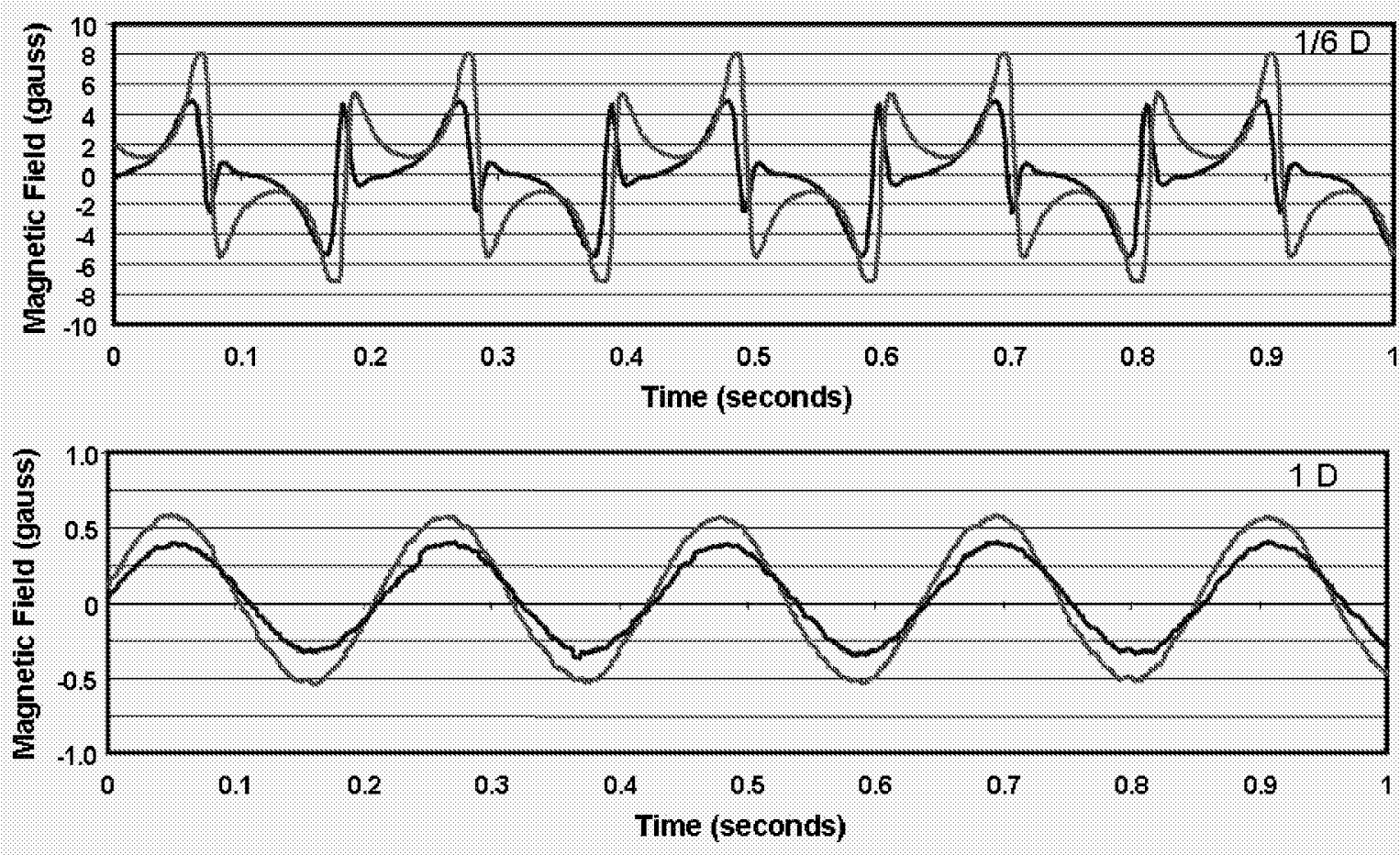

Figure 6. Axial and radial components of the magnetic field near the exciter (top) and one pipe diameter away (bottom).

Our calculations and experiments show that the magnetic field decay is exponential, as illustrated in Figure 7. The rate of decay in this 12-inch pipe is nominally an order of magnitude per pipe diameter. Experimental verification of these results was performed and superimposed on the modeling results. The rate of decay for theory and experiment matched well. The results of a comparison of signal levels for theory and experiment were within an order of magnitude, with the modeling providing higher values. 


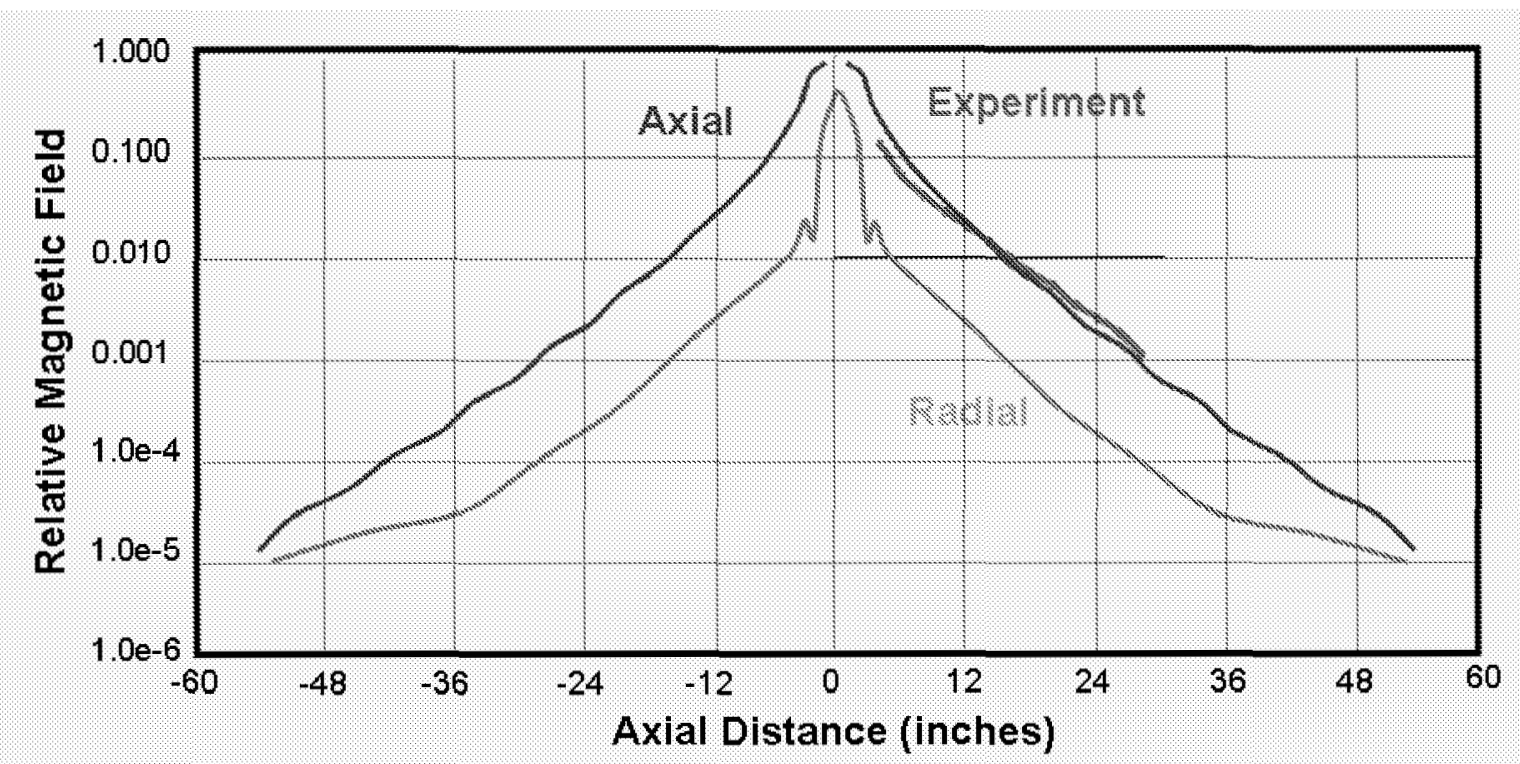

Figure 7. Axial decay of the currents flowing circumferential in the pipe wall.

The decay rate is related to both pipe diameter and number of poles. Three configurations were tested, a:

- 6 inch $(155 \mathrm{~mm}) 2$ pole

- 12 inch $(310 \mathrm{~mm}) 2$ pole, and

- 12 inch $(310 \mathrm{~mm}) 4$ pole.

The wall thickness of both pipe samples was nominally 0.375 inches $(10 \mathrm{~mm})$. It is assumed that the magnetic permeability and electrical conductivity of both samples are equal. The rotational frequency was 5 hertz. The rotation speed for the 4 pole unit was cut in half to keep the frequency of the inspection current equal to the other two configurations. The decay rates are show in Figure 8. The graphs show that the decay rate is similar for the 6 inch magnetizer with 2 poles and the 12 inch magnetizer with 4 poles; only the initial amplitude of the smaller diameter magnetizer is lower. The decay rate of the 12 inch magnetizer with 2 poles is nominally half of the other two. 


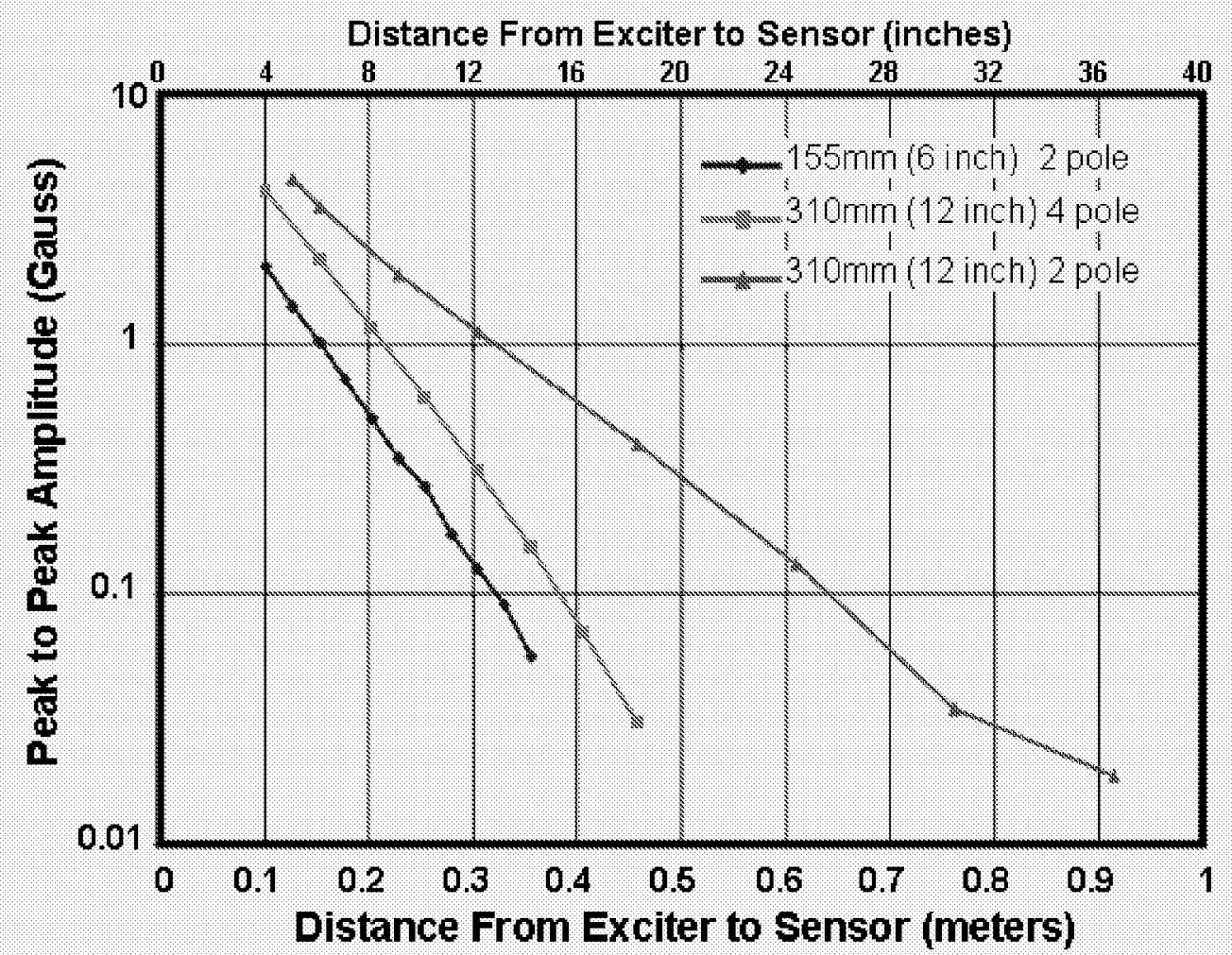

Figure 8. Current strength for 3 magnetizer configurations.

\section{Field Testing to Scan for Metal Loss Corrosion}

The wheeled prototype system shown in Figure 3 was pulled through a 12-inch diameter, 0.375inch thick pipe with machined metal loss defects. Data from both an axial and a radial Hall Effect sensor were recorded continuously as the tool was pulled smoothly through the pipe without stopping. The speed of the tool was nominally 3 inches per second. The rotation of the magnets was $300 \mathrm{rpm}(5 \mathrm{~Hz})$. A typical result is shown in Figure 9. The upper graph shows the unprocessed sinusoidal signals. The lower graph shows a tracing through the peak values. The axial component of the magnetic field increases at the metal loss area. The radial component increases before the metal loss area and then decreases after. While typical variations in conductivity and permeability of the pipe can affect amplitude, by detecting both the axial and radial signal, one can simplify detection. 

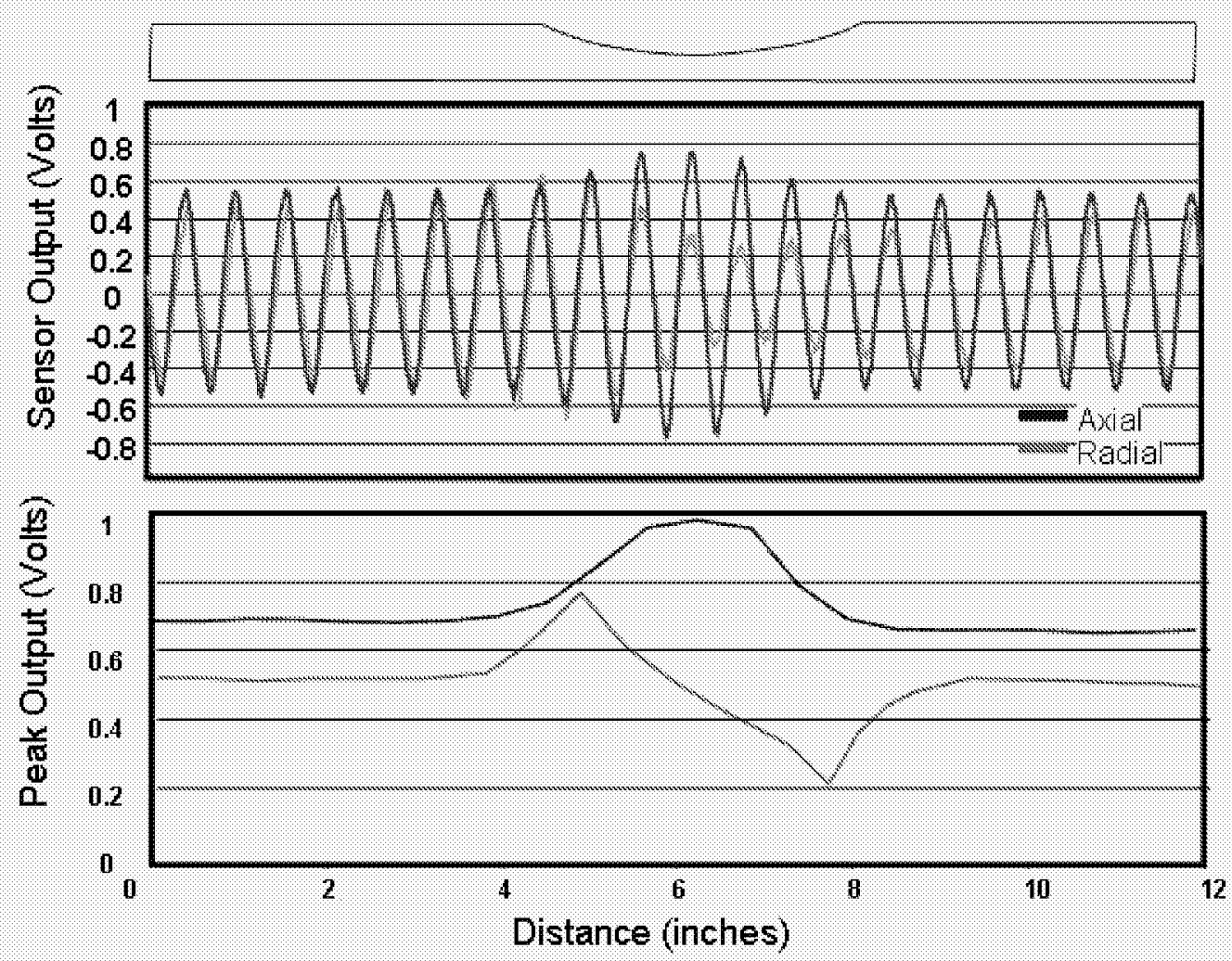

Figure 9. Typical signal from an area of pipe with a metal loss corrosion pit. 


\section{Results and Discussion}

\section{Detection of Metal Loss Corrosion}

The capability of this inspection method was demonstrated using blind benchmark tests conducted at Battelle's Pipeline Simulation Facility, where seven in-line inspection systems currently being developed under Department of Energy National Energy Technology Laboratory (DOE NETL) and Department of Transportation Research and Special Programs Administration (DOT RSPA) contracts were being tested. The rotating permanent magnet exciter was one of four sensor technologies used to examine the 12-inch diameter pipe for corrosion during a oneweek demonstration period in September 2004.

The detection and sizing characteristics of the defects ranged from simple to difficult. This helped to define both the current capability and future challenges for each of the inspection technologies. A list of specific distances and positions along the pipe were provided to each participant for reporting. The locations may or may not have had defects, enabling detection capability and false call rates to be assessed. It should be noted that each technology was at a different level of maturity, a fact which must be considered when evaluating results. Although a more mature technology may have provided better performance results in this assessment, over time a less mature technology may evolve to prove better suited for the needs of the pipeline industry.

In general, our inspection method found the larger defects and did not make any false calls. Also, the general characterization of size was encouraging. Specifically, we found defects with the following properties:

- MC09, which was 77\% deep and 2 inches long. This was characterized as deep and long.

- MC07, which was 45\% deep and 2.7 inches long. This was characterized as medium and long

- MC12, which was $48 \%$ deep and 3 inches long. This was characterized as small.

- MC15, which was 53\% deep and 1.5 inches long. This was characterized as medium and short.

- MC17, which was 72\% deep and 1.4 inches long. This was characterized as deep and long.

Additional details of these results can be found in Appendix B. Only one deep defect was not detected, MC05, which was 56\% deep and 1.2 inches long. The technique appears to be more sensitive to longer defects. This is important since length directly affects failure pressure. Thus, this method would have advantages over inspection technologies such as MFL, which are more sensitive to corrosion width and depth, possibly allowing narrow defects to go undetected. 


\section{Discussion}

The approach being developed by the Applied Energy Systems Group at Battelle has many advantages for inspection of nonpiggable pipelines. First, the two-pole permanent magnet can be closer to the pipe, producing stronger currents in the pipe wall than the commonly used concentric exciter coil. This arrangement will lead to stronger signals at the receiver. The twopole magnetizer has the form factor to pass rectangular obstructions such as plug valves. Each permanent magnet pole can be hinged to allow it to pass pipeline obstructions. For traditional concentric coil systems, the diameter of the exciter coil must be small enough to pass the largest obstruction. Although the RFEC technique works for exciter coils that have significant separation from the pipe, the induced currents in the pipe are locally weaker and more dispersed, causing a weaker signal at the receiver. Another way to boost signals at the receiver is to apply more current to the exciter coil, but this is not practical on autonomous vehicles. For the rotating permanent magnet exciter, the electrical power consumption should be dramatically less. Instead of continuously providing amps of power to energize the exciter coil, an efficient motor can be used to rotate the assembly. The motor will need a strong starting torque to overcome the static attraction force of the permanent magnets, but once the assembly is rotating, the power consumption is expected to reach a reasonable level.

Development of this novel approach to inspection energy generation began this year. The tool implementation tasks were accelerated to enable us to participate in the benchmarking study. As the tool used in the benchmarking was an early design for this method, we feel optimization of both the rotating magnetizer and sensor will improve results. We are using these results and finite-element modeling to increase signal to noise ratio to improve detection and sizing capability. Based on the benchmarking results, we are confident that a more robust system can be developed.

\section{Conclusions}

Battelle's Applied Energy Systems Group has built a rotating permanent eddy current exciter that produces strong magnetic fields at the receiver and has the potential to use less electrical power than coil-based eddy current systems such as remote field eddy currents (RFEC). The prototype unit produces strong eddy currents in the pipe wall. At distances of a pipe diameter or more, the currents flow circumferentially. These circumferential currents are deflected by pipeline defects such as corrosion and axially aligned cracks. Currents were detectable with a simple Hall Effect sensor at distances up to three pipe diameters away. Simulated corrosion defects were detectable in a pipe with a 12 -inch diameter and 0.375 -inch wall thickness. Numerous metal loss defects were detected during blind benchmark tests conducted at Battelle's Pipeline Simulation Facility.

This approach has many advantages for inspection of nonpiggable pipelines. The two-pole permanent magnet configuration has a form factor that would be capable of passing rectangular obstructions such as plug valves. The rotating magnet eddy current exciter, although in the early development stages, has the potential to perform as well as magnetic flux leakage and remote field eddy current inspection technology. 
Also in this period, assessment of the pulsed and rotating eddy current systems was performed. Benchtop implementations of each system was tested on similar defects. Since the rotating system has proven to be more adaptable to pipeline inspection, only this system will be carried into the second year of the sensor development. 
Appendix A

Pull Away Tests 


\section{Appendix A}

\section{Pull Away Tests}

The goal of the rotating magnetizer is to produce a uniform current of appreciable magnitude. The magnetic field in the pipe has two parts, each with distinct properties and effects. One part is the direct magnetic field from the strong permanent magnets. The second field is due to the current flowing in the pipe. Near the rotating magnets, the direct field from the magnet is dominant and produces a saddle-shaped alternating signal. Farther away from the magnets, magnetic field caused by the currents flowing in the pipe dominates. Figure A-1 shows the magnetic field at the inside surface of the pipe at distances ranging from close to the magnetizer to 2.5 pipe diameters away for a two-pole system in a 12 -inch pipe spinning at 5 hertz. The field due to direct field is negligible at distances greater than one pipe diameter (1D) and the measured signal is nearly sinusoidal. The field is also strong, on the order of a gauss. The currents are detectable at distances beyond 2 pipe diameters. Figure A-2 shows the magnetic field at the inside surface of the pipe at distances ranging from close to the magnetizer to 1.5 pipe diameters away for a four-pole system in a 12 -inch pipe spinning at 2.5 hertz. The signal levels at similar distances are not as strong. 

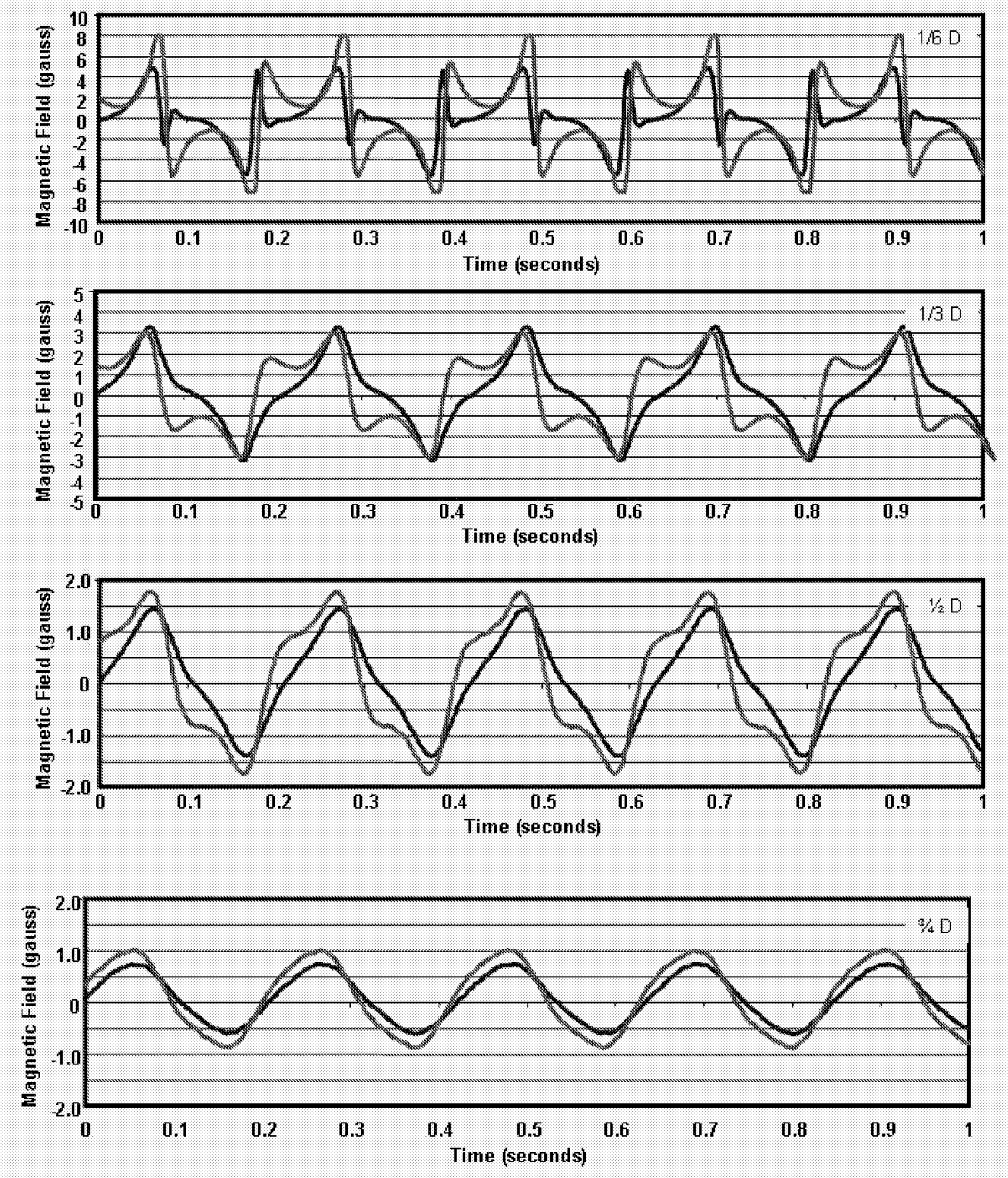

Figure A-1. Magnetic field at the inside surface of the pipe at distances ranging from close to the magnetizer to 2.5 pipe diameters away for a two-pole system in a 12-inch pipe spinning at 5 hertz. 

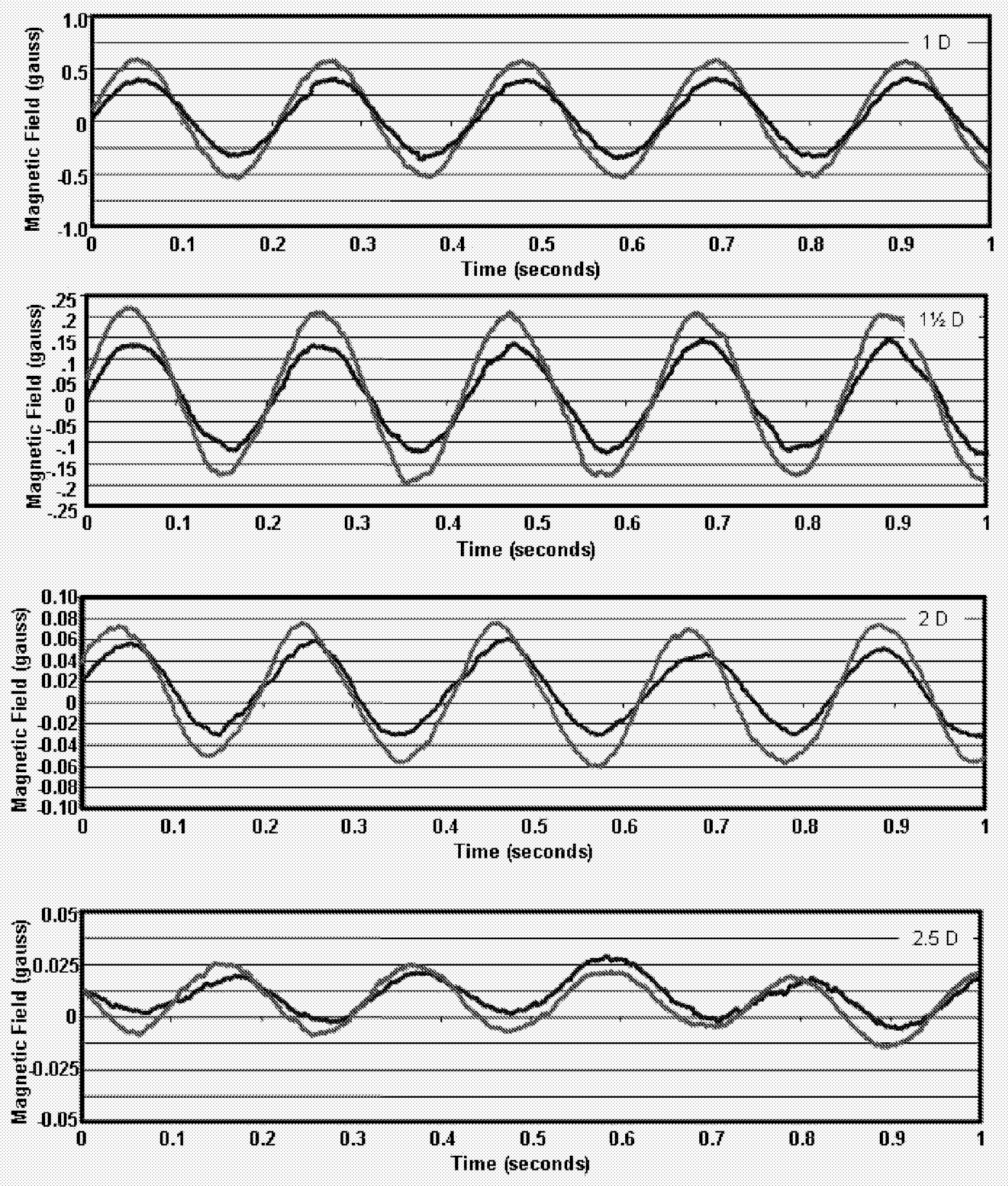

Figure A-1 (cont) Magnetic field at the inside surface of the pipe at distances ranging from close to the magnetizer to 2.5 pipe diameters away for a two-pole system in a 12-inch pipe spinning at 5 hertz. 

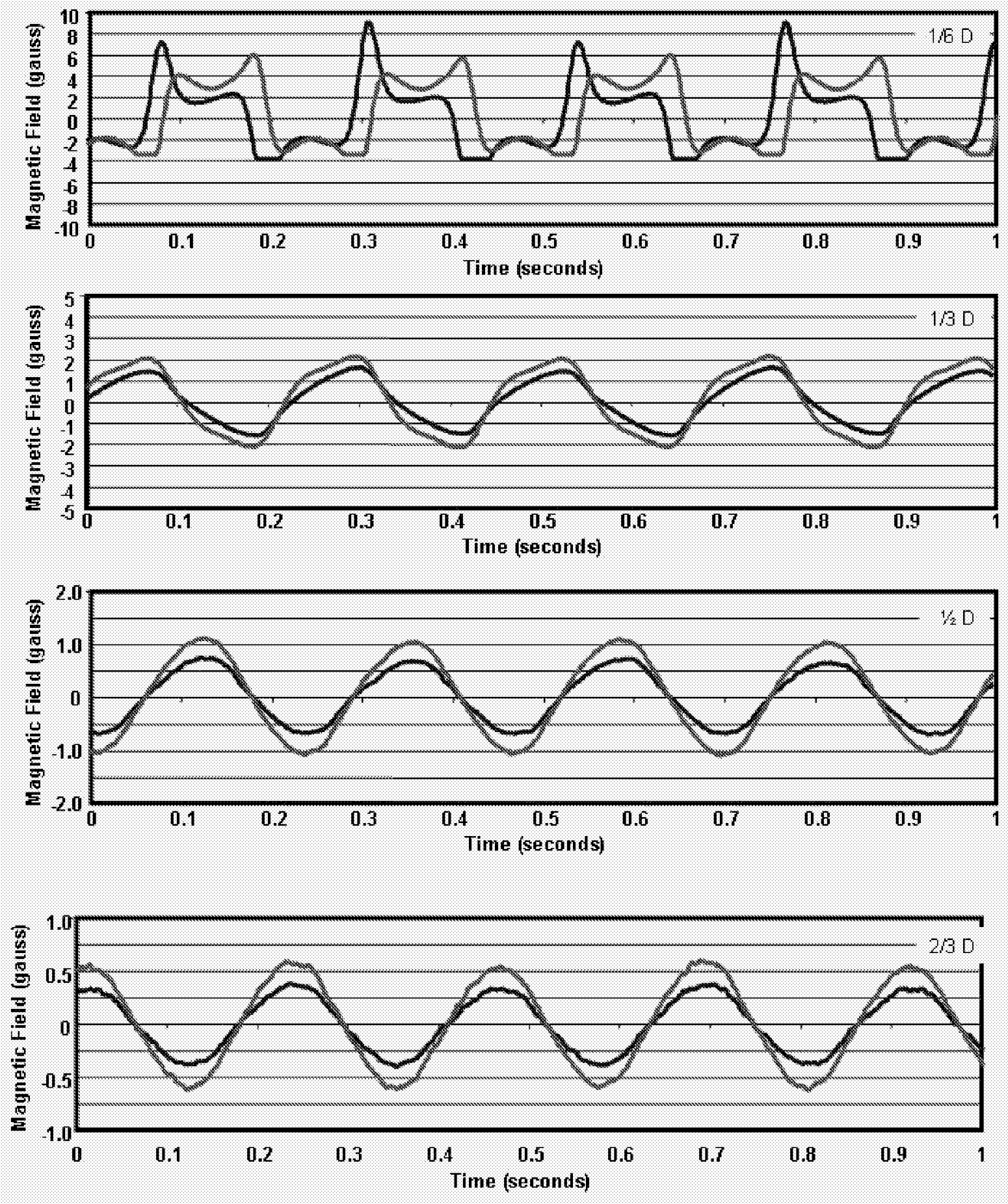

Figure A-2. Magnetic field at the inside surface of the pipe at distances ranging from close to the magnetizer to 1.5 pipe diameters away for a four-pole system in a 12 -inch pipe spinning at $2.5 \mathrm{hertz}$ 

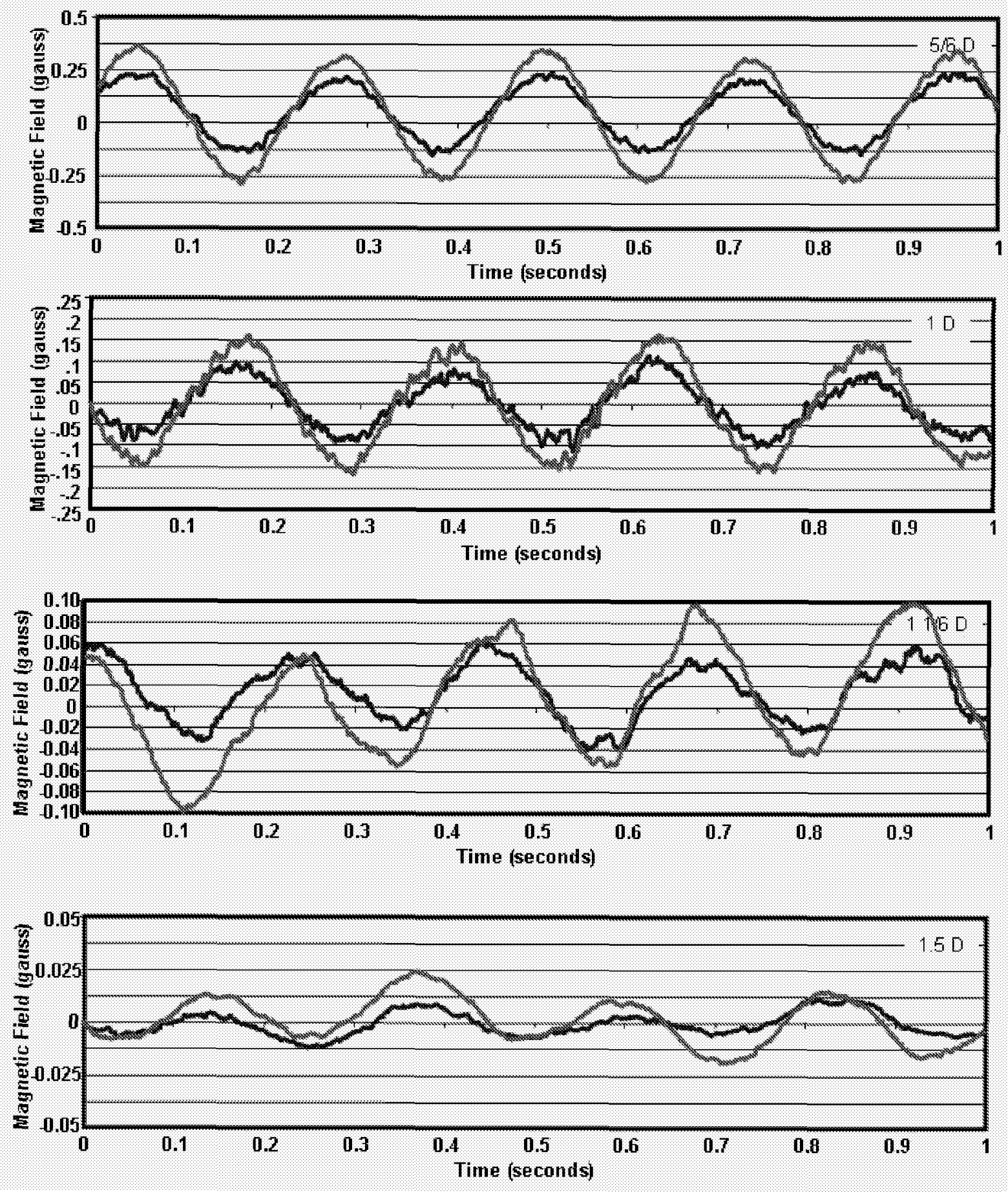

Figure A-2 (cont). Magnetic field at the inside surface of the pipe at distances ranging from close to the magnetizer to 1.5 pipe diameters away for a four-pole system in a 12-inch pipe spinning at 2.5 hertz 
Appendix B

Benchmark Tests at the Pipeline Simulation Facility 


\section{Appendix B}

\section{Benchmark Tests at the Pipeline Simulation Facility}

The NETL Gas Delivery Reliability Program develops innovative sensor systems that provide enhanced assessments of the status of transmission and distribution pipelines. This includes sensors to detect corrosion defects, stress corrosion cracking, plastic pipe defects, physical damage areas, gas content, gas contamination, and 3rd party intrusion near gas line right-ofways. A primary program goal is to develop ILI sensors that can be deployed remotely as part of an integrated robotic platform/sensor package. A sensor benchmark test was conducted at Battelle's Pipeline Simulation Facility (PSF) as a key step toward achieving this goal.

The report found on the NETL website provides a brief summary assessment of the demonstration test results ${ }^{1}$. The purpose of this assessment is to help identify promising inspection technologies best suited for further development as part of an integrated teaming effort between robotic platform and sensor developers. This document is not intended to provide a detailed analysis of each technology's performance or to rate their performance relative to one another.

Eight innovative sensor technologies were demonstrated at Battelle's PSF the week of September 13,2004 . The different technologies demonstrated their ability to detect pipeline corrosion, mechanical defects or stress corrosion cracking. In the blind benchmark tests, the rotating permanent magnet inspection method found the larger defects and did not make any false calls. Also, the general characterization of size was encouraging. Specifically, we found defects

- MC09, Figure B-1, which was 77\% deep and 2 inches long, characterized this as deep and long.

- MC07, Figure B-2, which was 45\% deep and 2.7 inches long, characterized this as medium and long

- MC12, Figure B-3, which was 48\% deep and 3 inches long, characterized this as small.

- MC15, Figure B-4, which was 53\% deep and 1.5 inches long, characterized this as medium and short.

- MC17, Figure B-5, which was 72\% deep and 1.4 inches long, characterized this as deep and long.

Only one deep defect was not detected, MC05, which was 56\% deep and 1.2 inches long. The technique appears to be more sensitive to longer defects. This is important since length directly affects failure pressure. This method would have advantages over inspection section

\footnotetext{
${ }^{1}$ Benchmarking Emerging Pipeline Inspection Technologies is available on the SCNGO homepage at www.netl.doe.gov/scngo/Natural\%20Gas/publications/t\&d/Benchmark\%20Emerging\%20Technologies\%20Final\%2 OReport.pdf
} 
technologies such as MFL which are more sensitive to corrosion width and depth, and narrow defects can go undetected.

Development of this unique approach to inspection energy generation began this year. The tool implementation tasks were accelerated to enable us to participate in the benchmarking study. As the tool used in the benchmarking was the initial design for this method, we feel optimization of both the rotating magnetizer and sensor will improve results. We are using these results and finite element modeling to increase signal to noise ratio to improve detection and sizing capability. With the benchmarking results, we are confident that a more robust system can be developed.

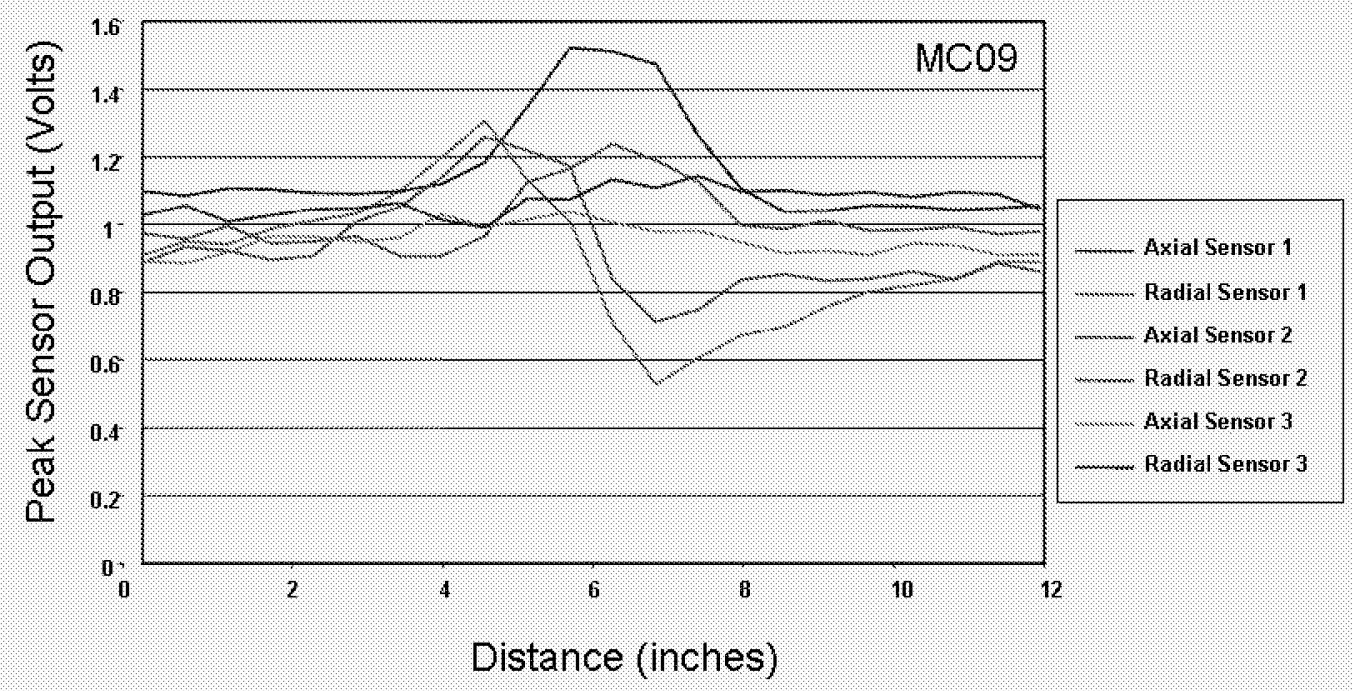

Figure B-1. Signal from corrosion anomaly $\mathrm{MC} 09$, which was 77\% deep and 2 inches long, char acterized this as deep and long 


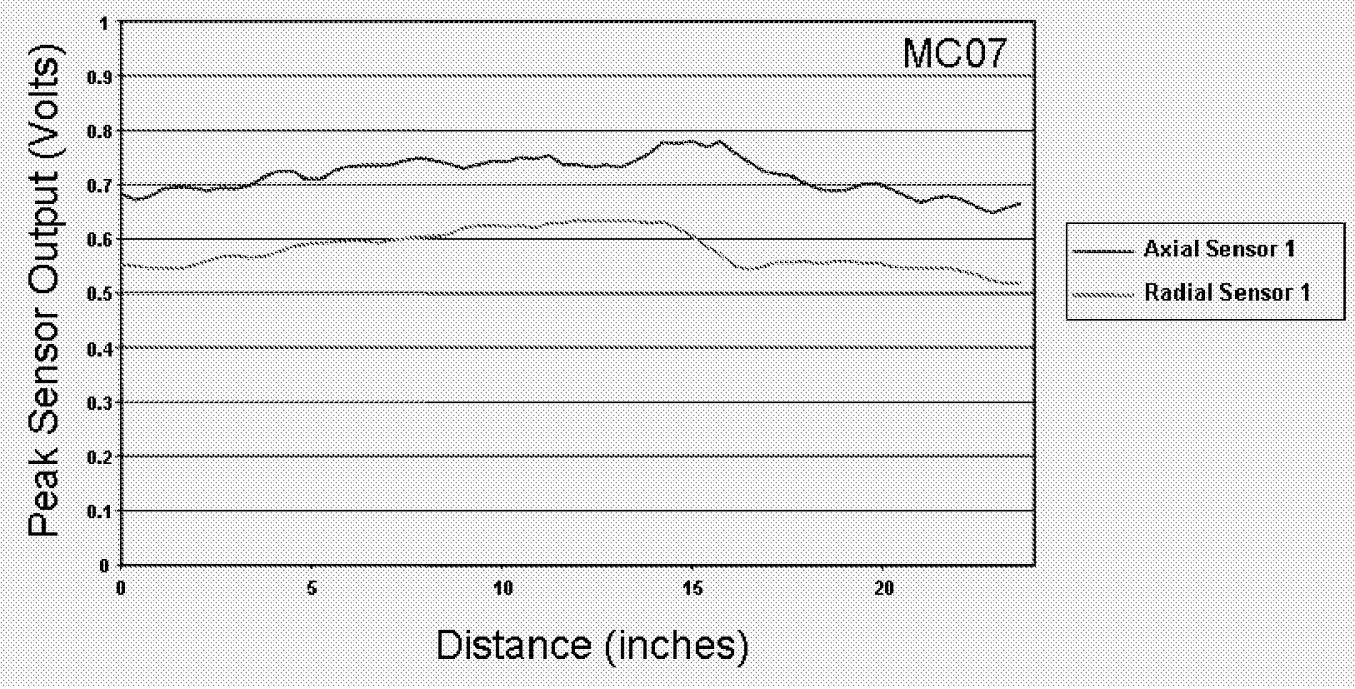

Figure B-2. Signal from corrosion anomaly MC07 which was 45\% deep and 2.7 inches long, character ized this as medium and long

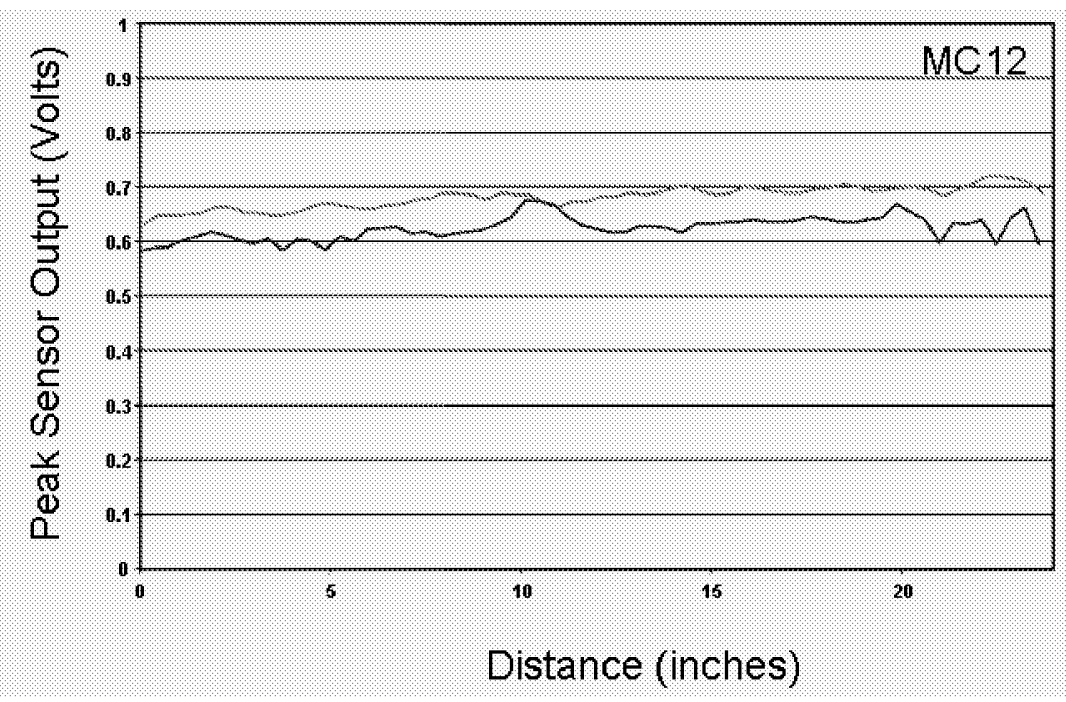

Axial Sensor 1

Radial Sensor 1

Figure B-3. Signal from corrosion anomaly MC23, which was 48\% deep and 3 inches long, characterized this as small. 

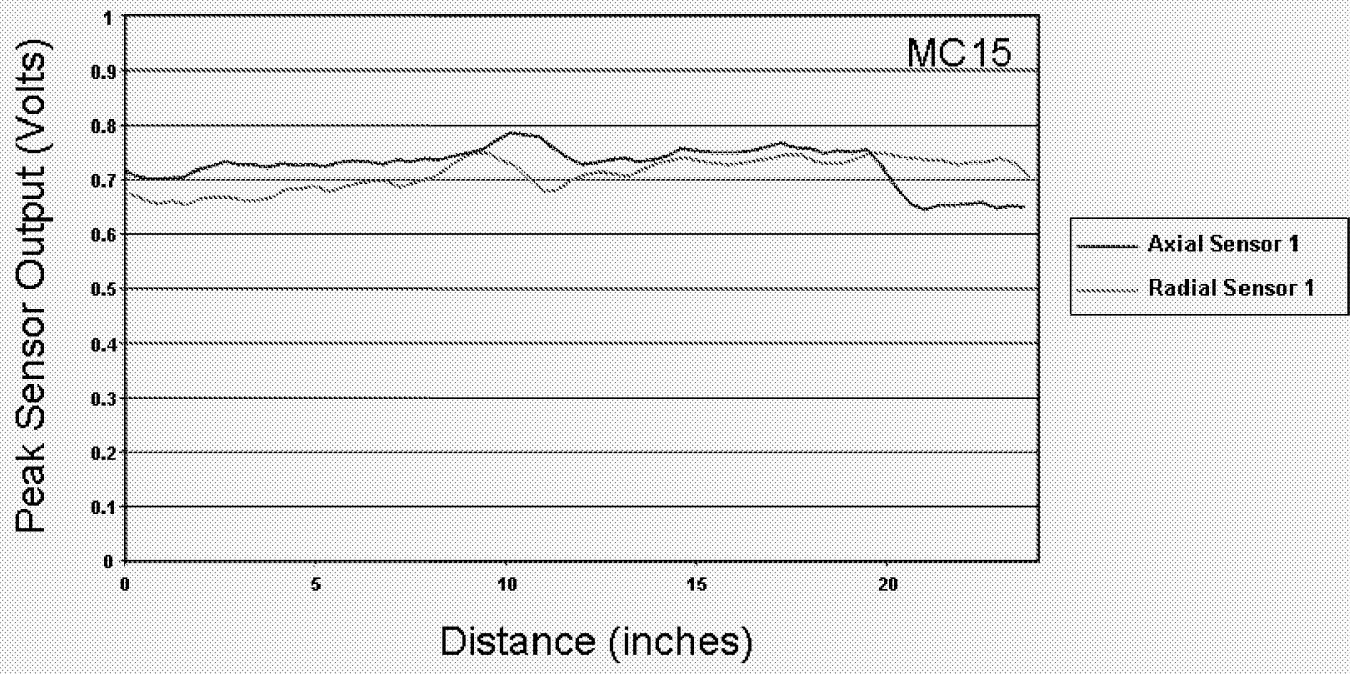

Figure B-4. Signal from corrosion anomaly MC15 which was 53\% deep and 1.5 inches long, characterized this as medium and short.

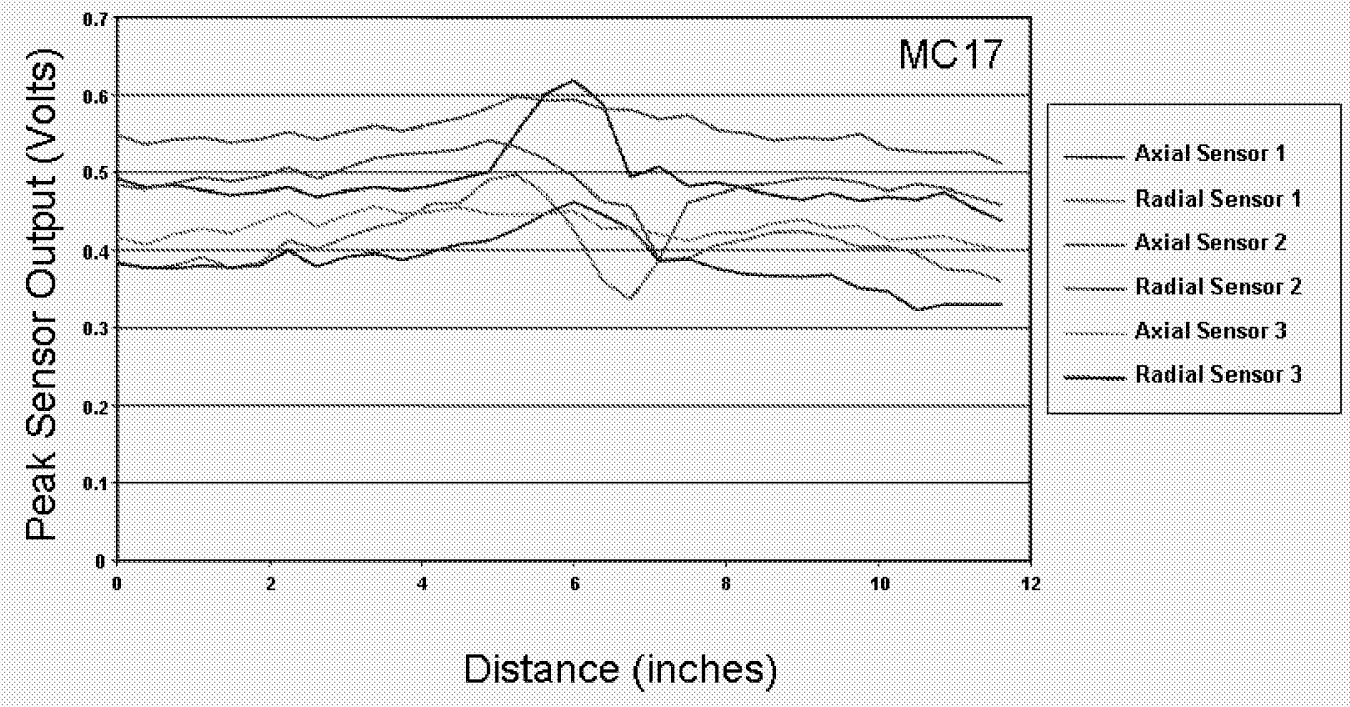

Figure B-5. Signal from corrosion anomaly MC17, which was $72 \%$ deep and 1.4 inches long, characterized this as deep and long. 